of disorders in man including such divergent diseases as viral hepatitis (A. J. Zuckerman, Immunopathology VI, edit. by P. A. Meischer, Schwabe, Basel, 1971, p. 436) and multiple sclerosis (F. Wolfgram et al., Multiple Sclerosis, Immunology, Virology and Ultrastructure, Academic Press, 1972). This is now the subject of intensive investigation in many laboratories.

Traditionally the tissue damage caused by viral infections has been explained by the ability of viruses to multiply in cells, thereby leading to cell injury or destruction. It is now becoming clear, however, that other mechanisms may be operating and that lesions may be caused by the immune response of the host to viral antigens and that the immune system itself may be disturbed by some viruses. Thus, certain viruses depress the humoral and cellular immune responses of the host, whereas other viruses may enhance the antibody response. Several viral antigens persist in the circulation and combine with specific antibody resulting in antigen-antibody complexes. These complexes may lodge in various sites, and in other instances combination with complement releases substances which cause tissue damage. Immunological injury mediated by antibodies may also result when new antigens produced by viruses on infected cell surfaces interact with specific antiviral antibody and complement (Memorandum: Virusassociated Immunopathology, Bull. Wld Hlth Org., 47, 25 ; 1972).

This concept of virus-associated immunopathology has recently been extended to temperate viral infections and it is possible that the ability to produce infectious immune complexes may be important in establishing chronic infections such as SSPE and hepatitis $B$ in man and lymphocytic choriomeningitis, aleutian mink disease and murine sarcoma and leukaemia in animals. Chronicity may be attributable to differences in the affinity of the antibodies produced, a factor which is partly under genetic control, perhaps on mechanisms linked to histocompatibility genes (W. F. Bodmer, Nature, 237, 139 ; 1972). It is difficult, however, to explain the relative rarity of SSPE, if it is caused by measles virus, in the face of the ubiquitous distribution of measles. What is needed is an animal model, but experimental inoculations of animals with different strains of SSPE agent have generally yielded divergent results. P. Albrecht and colleagues (J. Infect. Dis., 126, 154 ; 1972) have reported recently that a hamsterbrain-adapted strain of measles virus produced encephalitis in rhesus monkeys with morphological findings similar to those described in SSPE and quite unlike acute measles encephalitis in man. The principal property respon- sible for this potential of measles virus seems to be an aberrant intracellular replicative cycle of the virus which affects the maturation process at the cell membrane. Further understanding of temperate viral infections in humans thus seems likely in the near future.

\section{PHOTOPHOSPHORYLATION \\ Without Membranes}

from our Photosynthesis Correspondent IN 1954 it was discovered that isolated chloroplasts courd utilize light energy to make adenosine triphosphate (D. Arnon et al., Nature, 174, 394). This finding had important implications because it meant that chloroplasts could produce ATP as well as $\mathrm{NADPH}_{2}$ for driving $\mathrm{CO}_{2}$ fixation. Photophosphorylation is now a well documented phenomenon which can readily be detected with lamellae fragments obtained from chloroplasts which have been broken osmotically. It has even been shown to occur in small subchloroplast vesicles obtained either by sonication (R. E. McCarty, J. Biol. Chem., 244, 4292 ; 1969) or by treatment of inner lamellae with digitonin (N. Nelson et al., ibid., 245,$143 ; 1970$ ). The common feature of these phosphorylating systems was that they were dependent on the presence of an intact membrane and also they required the addition of electron carriers or acceptors. It now seems possible, however, that photophosphorylation can occur in the absence of both intact membranes and artificially supplied electron carriers.

McPhee and Brody (Proc. US Nat. Acad. Sci., 70, 50; 1973) report the striking observation that an acetone extract obtained from chloroplasts when layered on an aqueous surface will exhibit light-induced phosphorylation. The extract was obtained from particles derived by sonicating spinach chloroplasts, and was used to produce a monolayer at an air-water interface by compression to a surface tension of about 20 dynes $\mathrm{cm}^{-1}$. Electron microscopy indicated the films to be partly composed of small structured particles of about $200 \AA$ diameter at a concentration of one particle per $2 \times 10^{8} \AA^{2}$ of compressed surface. On illumination McPhee and Brody detected ATP production with quantum yields between 0.1 and 1 . The ability of the monolayer to catalyse photophosphorylation was dependent on maintaining a compression of at least 10 dynes $\mathrm{cm}^{-1}$ and could be doubled by adding phenazine methosulphate.

The artificial system was also found to induce additional esterification of inorganic phosphate not directly correlated with ATP formation. To explain their results $\mathrm{McPhee}$ and Brody argue that the phosphorylation observed may take place by a two-step reaction, one involving transphorylation between two ADP molecules to yield AMP and ATP followed by esterification of AMP to ADP.

Although McPhee and Brody have not used their data to discuss the chemical (E. C. Slayter, Eur. J. Biochem., 1, 31 ; 1967) or chemiosmotic (P. Mitchell, Biol. Rev., 41, 445 ; 1966) hypotheses their observations will undoubtedly be used by the "anti-chemiosmotic school" in attempts to dislodge Mitchell's elegant hypothesis.

\title{
Poly(A) Tract and Messenger RNa Ageing
}

THE function of the tracts of adenylic acid residues that are known to occur at the $3^{\prime}$ ends of many messenger RNA molecules remains a mystery, but experiments reported by Sheiness and Darnell in Nature New Biology next Wednesday (February 28) indicate that as messengers age in the cytoplasm of HeLa cells the length of the poly(A) tracts decreases; this may be a clue to their function.

Sheiness and Darnell analysed the poly(A) liberated by ribonuclease from populations of messenger RNAs in cytoplasmic extracts of HeLa cells fed pulses of ${ }^{3} \mathrm{H}$-adenosine and then actinomycin D. They conclude from such experiments, first, that the tracts of poly(A) in cytoplasmic messengers shorten as a result of metabolic ageing and, second, that this shortening occurs in the cytoplasm after the messengers have been transported from the nucleus. Furthermore, this timedependent shortening of the poly(A) tracts occurs when protein synthesis is blocked. It seems plausible to suggest therefore that the lifetime of a messenger may be determined by the length of the $3^{\prime}$ poly(A) tract and the rate at which the messenger loses adenylic acid residues. In other words, the function of poly(A) tracts of messengers may be to programme the lifetime of the molecules.

Also in Nature New Biology next week Gross et al. report the isolation from the polysomes of sea urchin embryos of a "9S" RNA fraction which stimulates a cell-free system from Krebs II mouse ascites cells to incorporate amino-acid. Fingerprint analysis of the product of in vitro translation indicates that it includes histones, and by comparing the stimulation of incorporation of tyrosine and tryptophan by 9S RNA Gross et al. conclude that their 9S RNA seems to be relatively free of contamination by messengers for proteins other than histones. 